\title{
32 Educational experiments of distance learning and reorganisation of mathematics education
}

\author{
Klaus-D. Graf \\ Freie Universität Berlin \\ Germany \\ Kiyoshi Yokochi \\ Yamanashi University \\ Kofu, Japan
}

\begin{abstract}
Rapid development of multimedia information technology makes possible the realisation of distance learning between two classes not only in same country but also in different countries. On the other hand, we expect that distance learning must play an important role in mathematics education throughout the world (Ito, 1996; Graf, 1995). In this connection, in Oct. 1995, the CCV-Educational System Study Group was organised in Japan for Distance Learning (DL) by Kiyoshi Yokochi. CCV is an abbreviation for Computer, Communication and Visual. At present, the Study Group has two subgroups, a Japan DL Subgroup and a German DL Subgroup chaired by K. Yokochi and Klaus-D. Graf respectively. In the following we will explain the progress and results of the experiments executed by the Study Group since 1995. The equipment for the experiments was provided by Mitsubishi Electric Corporation.
\end{abstract}

\section{Keywords}

Culture, distance learning, information technology, mathematics education, multimedia, teleconferencing. 
266 Information and communications technologies in school mathematics

\section{PURPOSE OF THE EXPERIMENTS}

The content of the experiments is teaching mathematics. The experiments are aimed at some reorganisation of mathematics education through DL. The aims of the experiments were as follows:

- initiating mathematical creativity of pupils;

- learning comprehensive uses of mathematics with other subjects;

- improving the mathematical scholarship of pupils;

- appreciating the mathematical cultural characteristics of each district or country;

- cultural exchanges between pupils of both classes.

\section{THE FIRST STAGE EXPERIMENT}

The experiments have been performed in three stages since Oct. 1995. In the first two stages, the following equipment (abbreviated as 'system') was installed in the class or in Mitsubishi Laboratory Centre (abbreviated as 'Mitsubishi Lab.') in Yokohama:

- Audio-Visual equipment (AV) for exchanges between classes through camera images and voice signals.

- Personal computers (PCs) and large (80 inch) displays on which PC or AV images were projected.

The two systems were connected by an ISDN line with a speed of 384kbps. The first stage experiment was preparatory (Yokochi, 1996, March). From Oct. 1995-March 1996, two types of DL were executed. The first type was between the Elementary School attached to Yamanashi University (abbreviated as Yamanashi) and the Elementary School attached to Yamagata University (abbreviated as Yamagata). Learning content was to make paper models using development in the third grade. The number of pupils of Yamanashi and Yamagata was 40 and 16 , respectively.

The second type of DL was between Yamanashi and Kanbe Elementary School (abbreviated Kanbe). The number of pupils in Yamanashi and Kanbe was $\mathbf{4 0}$ and 37, respectively. Learning content was 'speed of turtle' and 'speed of wind' in the third grade. Each type of DL consisted of 2 lessons of about 90 minutes. The system was installed in Yamanashi and the Mitsubishi Lab. Therefore, the teachers of both Yamagata and Kanbe had to come to the Mitsubishi Lab. with the videos of their own lessons and the pupils' works for each lesson. In the following, we will explain the first type of DL.

In the first lesson, Yamanashi's pupils showed Yamagata's teacher their original work (treasure box) and explained the developments of their work (see photo 1). 
Yamagata's teacher appraised the work and discussed it with them. On the other hand, Yamagata's pupils showed Yamanashi's pupils their original work (cars) and explained the developments of their work. Yamanashi's pupils appraised the works and discussed it with Yamagata's teacher. Two classes' methods of constructing development were considerably different. At the end of the lesson, both classes agreed to make the other class's paper models using developments embodying both classes' methods of constructing developments.

In the second lesson, Yamanashi's pupils showed Yamagata's teacher their original work (cars) and explained the developments of the work (see photo 2). Yamagata's teacher appraised the work and discussed it with them. After the lesson, Yamagata's pupils' work was shown to Yamanashi's pupils.

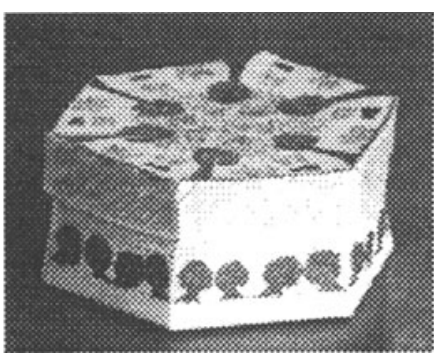

Photo 1 Example of a treasure box

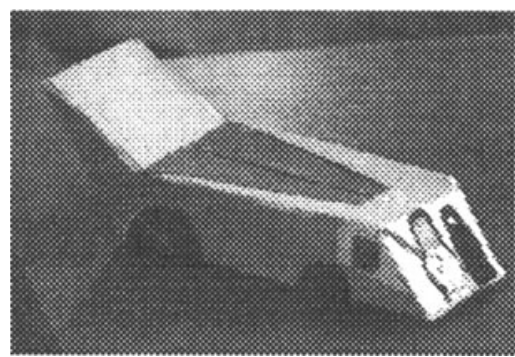

Photo 2 Yamanashi pupil's racing car.

Through the above experiment, we found the following:

- Many pupils endeavoured to use their original method of development with reference to the other class's method.

- Many pupils endeavoured to make their original paper model not only with reference to original works of the other class's pupils, but also with much better idea than any works of the other class's pupils.

We now comment on the second type of DL. The experiment showed that each class's pupils found several original methods for measuring speed and put them practice under impetus from the other class's methods.

The above results of the 1st stage experiment suggest the attainment of our first three purposes of using DL.

\section{THE SECOND STAGE EXPERIMENT}

After good results from the first stage experiment, the second stage experiment was performed in April-Oct. 1996 (see Yokochi, 1996, October). It consisted of four types of DL between Yamanashi and Yamagata. The system was installed in both classes. Learning content, the number of the lessons and the pupils of each type were as follows: 
- The first type: Location and co-ordinates on a plane in the first grade. Three lessons of about 60 minutes. Yamanashi's number: 40, Yamagata's number: 40.

- The second type: Modelling with clay and curved surface in the first grade. Three lessons of about 60 minutes. Yamanashi's number: 40, Yamagata's number: 40.

- The third type: Using letters in solving practical problems in the fourth grade. Two lessons of about 60 minutes. Yamanashi's number: 37, Yamagata's number: 8.

- The fourth type: Making picture-cards, curved line relating to curvature and properties of circle in the fourth grade. Three lessons of about 60 minutes. Yamanashi's number: 37, Yamagata's number: 8.

In the following, we explain some results of the second type and the fourth type.

\section{The second type}

In the 1st lesson, Yamanashi pupils showed Yamagata pupils their original work (masks) with pasted papers, like a fat-faced woman's mask or a distorted male mask (see photos 3 and 4). The models of masks made of clay, addressing their attention to curvature of mask's surface. Then, they explained to Yamagata pupils the mathematical meaning of curvature with circles using the sections of a fat-faced woman's mask. On the other hand, Yamagata pupils showed Yamanashi pupils their original works of animals with clay (see photo 5). In each animal, a wire was inserted, which was shaped by each pupil with attention to the skeleton of the animal. Then they explained the skeleton of a horse and its function. The pupils of both classes discussed continually the above things with each other. At the end of the lesson, both classes agreed upon the following. At the third lesson after 25 days, Yamanashi pupils and Yamagata's show lively sportsmen and animals with clay, respectively.

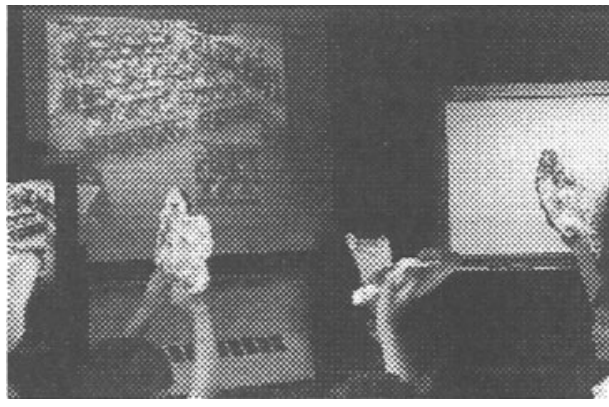

Photo 3 At the beginning of lesson, Yamanashi pupils show Yamagata's their masks.

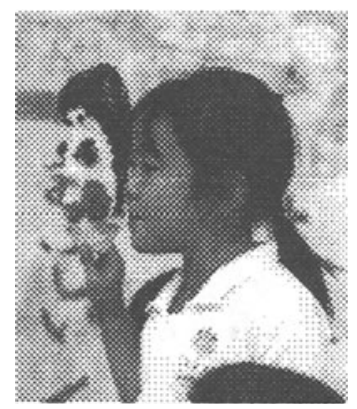

Photo 4 A Yamanashi pupil explains her mask to Yamagata pupils. 
In the second lesson, the day after the first one, both classes observed lively sportsmen and animals through videos or animation. Then, both classes' pupils pictured the shapes of their work to make with clay and showed them using gestures made by hands and feet. And both classes' pupils discussed their gestures with each other. In the third lesson, as promised, both classes' pupils showed their original work and explained their difficulties with the work.

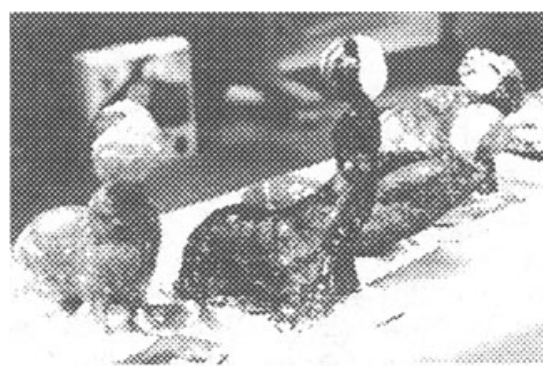

Photo 5 Yamagata's pupils' works (sea lions).

Through the above experiment, we found the following:

- Pupils understood well enough the curvature of the curved surfaces and characteristic curvatures according to the animal's skeleton.

- All the pupils completed excellent lively works by their own ideas, which were far above their works in the first lesson.

The above results demonstrated attainment of the first three purposes of DL.

\section{The fourth type}

Before the first lesson, each group of about seven pupils in both classes made their original picture-cards based on folktales in their districts. Along the contours of each picture of the cards, woollen yarns of several colours were stuck. The procedure of the above work was as follows.

Each group selects a folktale. Picture-cards based on the above folktale are drawn. Contours of each picture are divided into several parts, along which woollen yarn of specified colour is to be stuck. Each part is divided into a number of sections which approximates to an arc of a certain circle. For each section, the centre of the circle is constructed by one of following two tools and the radius of the circle is measured. Yamanashi's tool is a wide ruler $14 \mathrm{~cm}$ long made of a

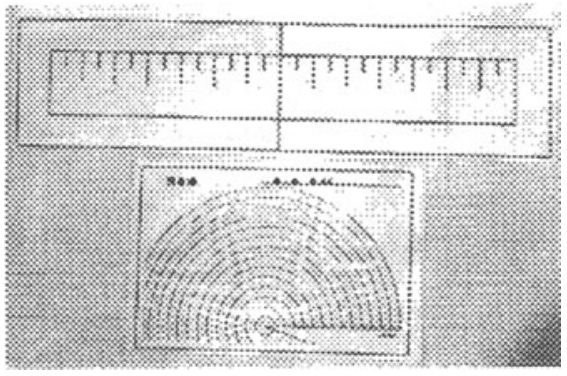

Photo 6 Two tools. transparent sheet, on which its perpendicular bisector is notched (see photo 6). Using it, pupils could easily draw the perpendicular bisector of a segment. Yamagata's tool is a disc of $14 \mathrm{~cm}$ radius made of a transparent sheet on which concentric circles are notched, each with a radius of $0.5 \mathrm{~cm}$ (see photo 6). Using it, pupils could easily find the centre of a circle from 1st arc. 
For each section, its central angle is measured with a protractor. Curved lengths of each section are calculated with a calculator, using the radius and central angle. The length of each part is calculated with a calculator. Woollen yarn of the above length is given from their teacher and is stuck along the contour of the picture.

In the first lesson, both class's groups showed their original work (picture-cards) to each other and discussed the work. Then, Yamagata pupils introduced their tool to Yamanashi's. They explained how to use it, the principle of the tool, and the curvature of the curved lines. Then both class's pupils measured curvatures in several familiar curved lines found in their classes.

In the second lesson, one group of Yamanashi showed their original picture-cards based on a folktale. Then, Yamanashi pupils introduced their tool to Yamagata's

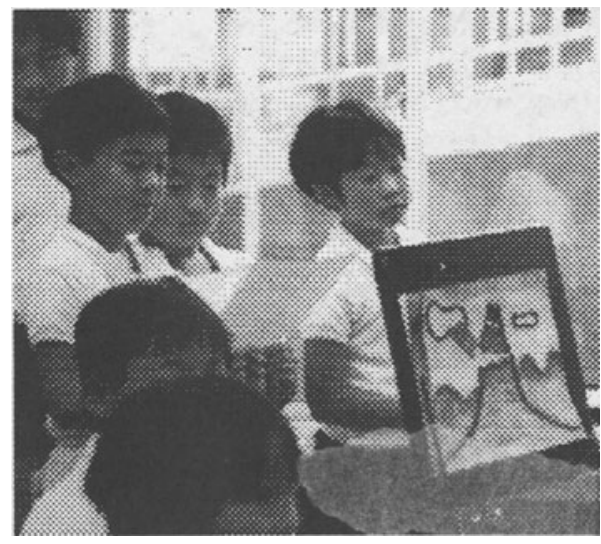

Photo 7 Yamanashi pupils explain scenes of their picture-cards. and explained how to use it and the principle of the tool. At the same time, they explained several properties of the circle using a computer. At the end of the lesson, the both classes agreed to show their original work (picturecards) with the aid of two tools at the third lesson after 17 days.

In the third lesson, Yamanashi's groups showed several picturecards of famous folktales and Yamagata's group showed picture-cards of the principal sights in their district (photo 7).

Through the above experiment, we found the following:

- Pupils understood well enough many properties relating to the circle and came to apply them to several real problems.

- Pupils understood well enough mathematical meaning of curvature and came to apply to drawing pictures or other things.

- In the third lesson, pupils completed excellent bold works, which were far above the standard of the first lesson.

- Pupils came to appreciate folk culture not only in their district but also in the other class's district.

The above results demonstrated the attainment of all of the purposes of DL. 
In the second stage experiment, before and after each lesson, pupils took several tests relating to the purposes of DL. And pupils' behaviours and work were analysed in the lights of the purposes. Those data proved the satisfactory attainment of the purposes. At this point, we want to give the following additional comments. In all types of experiment, we worked hard and long from preparation to the final lesson. Good results from DL are the reward for the work of DL's members.

On the other hand, we need to find a concise procedure for DL, following which many teachers may readily execute DL.

\section{Supplementary Experiment using ISDN at 128kpbs}

ISDN lines with a speed of $128 \mathrm{kpbs}$ are becoming a common standard. And they are much cheaper than ISDN at 384kpbs. Therefore in Jan. and Feb. 1997, two types of DL using ISDN at 128kpbs were performed between Ashizawa Elementary School (abbreviated Ashizawa) and Kanbe. The number of pupils of Ashizawa and Kanbe is 37 and 8, respectively. Some of the results of the experiment were as follows (Yokochi et al., 1997; Dasai et al., 1997).

- The behaviour of groups of three or four pupils can be distinguished. However, individual faces in the group are indistinguishable.

- If the lessons meet the next two qualifications, we can expect quite good results relating to the purposes of DL:

both classes are keenly enthusiastic in DL;

learning methods of both classes exert their ingenuity for DL.

\section{THE THIRD STAGE EXPERIMENT BETWEEN GERMANY AND JAPAN}

Based on the positive results of the Japanese experiments the authors organised four DL lessons between a Japanese elementary school attached to Yamanashi University in Kofu and a German one in Berlin. In addition to the five purposes already mentioned, two more became relevant: (1) practice of interaction situations influenced by constraints like different languages, and (2) the acknowledgement of different traditions and attitudes towards problem solving (Graf, 1995). In addition, special problems had to be solved for these joint international DL sessions: time difference, languages, teaching methods, and quality of moving pictures.

The two classes were from the fifth grade, 20 pupils in Berlin and 38 in Yamanashi. The German class had worked on rectangular patterns before, the Japanese class on stripe patterns. The DL sessions covered demonstrations of individual pupils' work and teachers' distance teaching about the mathematical background of this work (see photo 8). The engagement of the Japanese pupils 
was more intuitive, and that of the German pupils more systematic, including classification of transformations in a group table.

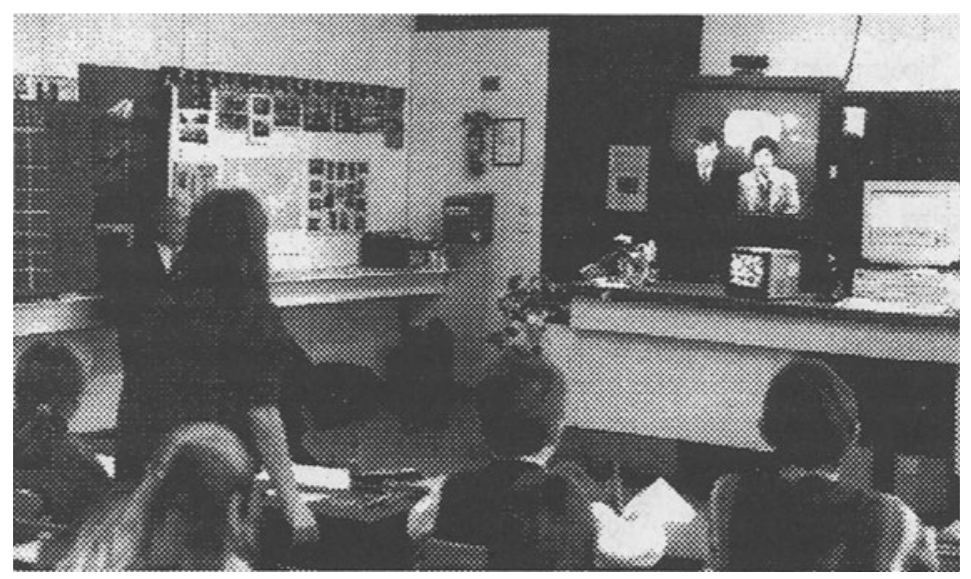

Photo 8 The classroom in Berlin.

While we are still far from reaching any general conclusions, some interesting observations can be made. The pupils took great interest in the project. They were planning with great zeal how to explain their results to the pupils in the other country. They also developed a very general interest in the other class environment and country and they discussed the project with other pupils, teachers and parents.

In the sessions they were extremely attentive and ready to perform. Without any problems in using the new technology they put direct questions to their peers in the other country, forgetting about the distance. They patiently waited for translations and showed interest and understanding of a different approach to solving problems, e.g., when aspects of art or tradition were included.

There are, however, two major obstacles to continuing the international version of the project. These are the high cost of equipment and communication lines and the enormous extra work which has to be done by the teachers when preparing these DL lessons.

\section{CONCLUSION}

The first and second experiments and the supplementary experiment were completed. The third stage experiment is in progress; first observations are 
reported above. Now, we will be able to round off those experiments with the following conclusions.

- The second stage experiments demonstrated that DL between 2 classes in the same country may attain the five purposes of DL. The third stage experiment has several negative conditions, that is, using ISDN at $128 \mathrm{kbps}$, large time differences, very novel content and so on. Therefore we expect the attainment of the seven purposes of DL between any different countries will bring about the same results as in the second stage experiment.

- The first five purposes accord with our needs of reorganisation of mathematics in the future from the following points of view-therefore, DL may be expected to do much for the reorganisation of mathematics education needed in future:

-To learn mathematics comprehensively with other subjects through solving real problems.

- To learn mathematics through mathematical activity with one's hands and feet.

-To learn higher level mathematics in this developing society with rapidity.

-To learn mathematics following one's originality and creativity.

- To learn mathematics in corporate with distant pupils in this expanding society.

-To learn mathematics in corporation with foreign pupils in this international society.

- In order to get good results from DL, it is very important to develop learning contents and learning methods for DL in close international co-operation.

- DL can be performed between any two classes inside and outside the country. In other words, any pupils may learn mathematics in co-operation with any pupils all over the world. We expect more and more pupils in the world to have the above chance.

- Our DL sessions were successful since we defined mathematical goals first and then used information and communication technology as a medium, not the other way round.

\section{REFERENCES}

Dazai, T., Koizumi, H. and Yokochi, K. (1997). A Collaborative Distance Learning System based on CSCW, Proceedings of DMS' 97 (Workshop on Distributed Multimedia Systems), Vancouver, Canada, 180-187.

Graf, K-D (1995). Promoting interdisciplinary and intercultural intentions through the history of informatics. In D. Watson, and D. Tinsley (eds.) Integrating Information Technology into Education. London: Chapman \& Hall, 139-150.

Ito, T. (ed.) (1996). Technology in the 21st Century. OHM Press. 
Yokochi, K. (ed.) (1996, March). Report of the First Stage Experiment by CCV Educational System. Mitsubishi Electric Company.

Yokochi, K. (ed.) (1996, October). Report of the Second Stage Experiment by CCV Educational System. Mitsubishi Electric Company.

Yokochi, K., Moriya, S., Okuyama, K. and Kuroda, Y. (1996, 97). Report of Experiments of Distance Learning by CCV Educational System New Education and Computer, 11, 5-7. Tokyo: Gakken.

Yokochi, K., Moriya, S. and Kuroda, Y. (1997). Experiment of DL using ISDN with 128kbs,. New Education and Computer, 6, 58-83.

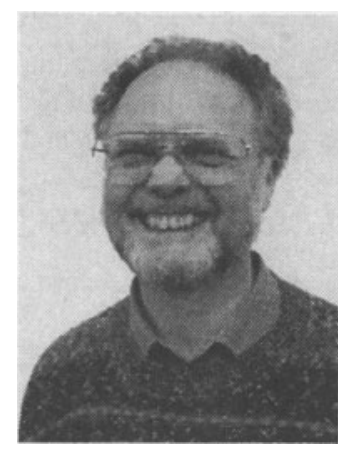

Klaus-Dieter Graf studied mathematics, physics and education in Erlangen, Berlin, Muenchen, Tuebingen and Mainz between 1957 and 1966. He was a research assistant at the University of Illinois and the California Institute of Technology in 1963 before taking his $\mathrm{PhD}$ in Mathematics at University of Mainz and becoming Assistant Professor in Cybernetics. He became Professor in Mathematics and Mathematics Education in 1971, and subsequently in cybernetics, computer science and computer science education at the Freie Universitaet Berlin. In 1987 he was visiting professor at the National Central University in Taiwan. Klaus-Dieter Graf specialises in applications of IT in mathematics and mathematics education, history of computer science, and international and intercultural co-operation. He is an active member of IFIP working groups and ICMI activities.

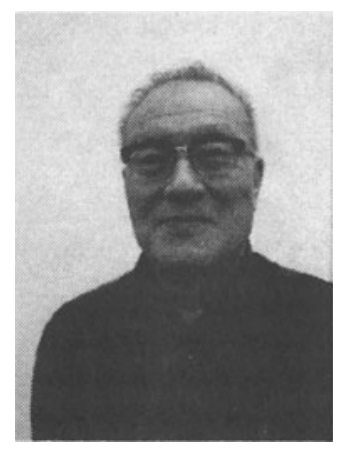

Kiyoshi Yokochi graduated from the Department of Mathematics at Tokyo Bunrika (Liberal Arts and Science) University in 1945. He held professorial posts at Wako University, Yamanashi and Tokai Universities before becoming Guest Professor at Beijing Normal University in 1985 . He has been President of the Mathematical Education Society of Japan since 1986, organiser of the Five Nations Conference on Mathematics Education since 1986 and organiser of the International Conference 'Cultural History of Mathematics' since 1991. His special fields are mathematics, mathematics education, informatics, cultural history of mathematics and kindergarten education. 